\title{
FIR Filter Design Using Distributed Maximal Flatness Method
}

\author{
Marek Blok
}

\begin{abstract}
In the paper a novel method for filter design based on the distributed maximal flatness method is presented. The proposed approach is based on the method used to design the most common FIR fractional delay filter - the maximally flat filter. The MF filter demonstrates excellent performance but only in a relatively narrow frequency range around zero frequency but its magnitude response is no greater than one. This ,passiveness" is the reason why despite of its narrow band of accurate approximation, the maximally flat filter is widely used in applications in which the adjustable delay is required in feedback loop. In the proposed method the maximal flatness conditions forced in standard approach at zero frequency are spread over the desired band of interest. In the result FIR filters are designed with width of the approximation band adjusted according to needs of the designer. Moreover a weighting function can be applied to the error function allowing for designs differing in error characteristics. Apart from the design of fractional delay filters the method is presented on the example of differentiator, raised cosine and square root raised cosine FIR filters. Additionally, the proposed method can be readily adapted for variable fractional delay filter design regardless of the filter type.
\end{abstract}

Keywords-digital filter design, fractional delay filter, distributed maximal flatness, differentiator, Nyquist filter, variable fractional delay.

\section{INTRODUCTION}

$\mathbf{P}$ RACTICALLY each digital signal processing algorithm requires digital filters. In this paper a new method allowing for design of finite impulse response (FIR) filters is presented. The proposed approach, called distributed maximal flatness (DMF), is the generalization of maximal flatness (MF) method. It has been originally developed for FD filter design [1] but as we demonstrate it in this paper, the proposed design method can be used to design any filter with optional fractional delay. We will demonstrate the method on examples of several special filters: fractional delay (FD) filter [2], differentiator [3] or Nyquist filters [4].

First from the mentioned above group of filters, is the FD filter which offers fine delay adjustment. This filter is required when delaying signal by an integer number of sampling periods delay is too coarse. The frequency response of the ideal fractional delay (FD) element with the total delay $\tau_{d}$ is following [2]

$$
H_{\mathrm{FD}}(\omega)=e^{-j \omega \tau_{d}}
$$

Since the impulse response corresponding to this frequency response

$$
h_{\mathrm{FD}}[n]=\operatorname{sinc}\left(n-\tau_{d}\right)
$$

is infinite and non-causal, in practice the delay element needs to be implemented using finite order causal FIR or IIR (infinite

M. Blok is with Faculty of Electronics, Telecommunications and Informatics, Gdańsk University of Technology, 11/12 G. Narutowicza Street, 80-233 Gdańsk Wrzeszcz, Poland (e-mail: mblok@eti.pg.gda.pl). impulse response) filter [2], [5]-[8]. In this paper we will only consider FIR filters. For these filters the total delay $\tau_{d}$ of the FD filter can be split into its integer $D$ and fractional $d$ part or into bulk $\tau_{N}=(N-1) / 2$ and net $\varepsilon$ part

$$
\tau_{d}=D+d=\tau_{N}+\varepsilon
$$

where $d \in[-1 / 2,1 / 2)$. With the assumption that the net delay $\varepsilon \in[-1 / 2,1 / 2)$, the net delay $\varepsilon$ is equal to the fractional delay $d$ for odd $N$. For even $N$ the fractional delay $d=\varepsilon-\operatorname{sign}(\varepsilon) / 2$ and has discontinuity at $\tau_{d}=\tau_{N}$.

The ideal impulse response of the digital differentiator is following

$$
H_{\mathrm{dif}}(\omega)=j \omega
$$

The most commonly used Nyquist filter is the raised cosine (RC) filter with the frequency response

$$
H_{\mathrm{RC}}(\omega)= \begin{cases}1, & 0 \leq \omega \leq \omega_{\mathrm{p}} \\ \cos ^{2}\left(\beta\left(\omega-\omega_{\mathrm{p}}\right)\right), & \omega_{\mathrm{p}}<\omega<\omega_{\mathrm{s}} \\ 0, & \omega_{\mathrm{s}} \leq \omega \leq \pi\end{cases}
$$

where $L$ is the symbol period in sampling intervals, coefficient $\beta=L /(4 \alpha), \alpha$ is the roll-off factor which determines lower and upper frequencies of the transition band $\omega_{\mathrm{p}}=L(1-\alpha) / 2$ and $\omega_{\mathrm{s}}=L(1+\alpha) / 2$. In communications systems the RC filter is split into two square root raised cosine (SRRC) filters. One is used as the shaping filter in a transmitter and the other as the matched filter in a receiver, both with squared frequency response of the $\mathrm{RC}$ filter

$$
H_{\mathrm{SRRC}}(\omega)=\sqrt{H_{\mathrm{RC}}(\omega)}
$$

Additionally, similarly to the FD filter, each of these filters is capable of introducing fractional delay, which allows for replacement of a multistage cascade of filters, zero-phase and FD filters, with a single optimized filter [3]. To obtain a filter with fractional delay, the ideal frequency response of the prototype zero-phase filter has only to be multiplied by the ideal frequency response of the FD filter

$$
H_{\mathrm{id}, \mathrm{FD}}(\omega)=H_{\mathrm{id}}(\omega) H_{\mathrm{FD}}(\omega)
$$

Like for the FD filter, ideal impulse responses of aforementioned filters also are infinite and non-causal and need to be approximated with finite order filter. In this paper we are investigating the approximation with the FIR filter of the length $N$ with the frequency response

$$
H_{N}(\omega)=\sum_{n=0}^{N-1} h[n] e^{-j \omega n}
$$

where $h[n]$ is the impulse response of the FIR filter. 
The performance of the designed FIR filter can be assessed based on magnitude response approximation error

$$
\operatorname{MAE}(\omega)=\left|H_{N}(\omega)\right|-\left|H_{\text {id }}(\omega)\right|
$$

and group delay error

$$
\operatorname{GDE}(\omega)=\tau_{\text {grp }}(\omega)-\tau_{\text {id }}(\omega)=\tau_{\text {grp }}(\omega)-\tau_{d}
$$

where group delay

$$
\tau_{\mathrm{grp}}(\omega)=-\operatorname{Im}\left(\frac{H_{N}^{(1)}\left(\omega_{k}\right)}{H_{N}\left(\omega_{k}\right)}\right)
$$

$H_{N}^{(i)}(\omega)$ denotes the $i$-th, here first, derivative of the frequency response $H_{N}(\omega)$.

Often instead of using two aforementioned error responses a single characteristic, the complex approximation error is used

$$
E(\omega)=H_{N}(\omega)-H_{\mathrm{id}}(\omega)
$$

In derivations it is often more convenient to use the modified error with removed phase component related to the bulk delay $\tau_{N}$

$$
\hat{E}(f)=E(f) e^{j \omega \tau_{N}}=\hat{H}_{N}(\omega)-\hat{H}_{\mathrm{id}}(\omega)
$$

For example in the minimax filter design [2], [9], [10] the maximum value of the magnitude of complex approximation error (12) is minimized in given approximation band, while for least squares (LS) filters [2] the energy of this error is minimized. Also the maximally flat (MF) FD filter design, presented in more detail in the next section, is based on the same error function.

\section{MF FD FILTER DESIGN}

The MF FD filters [2], [11]-[14] have very narrow band of good approximation located around zero frequency but their main advantage is their passivity [15], which means that their magnitude response at all frequencies is equal to or less than one. This property is very important if such a filter has to be used in a feedback loop. The novel DMF filter design method investigated here is the generalization of MF filter design. In the proposed approach the design equations are based, like in MF approach, on complex approximation error but the iterative optimization can be based also on different error functions. Additionally, a weighting function can be applied, which makes design more flexible.

The MF FD filter is characterized by maximal flatness forced at frequency $\omega=0$. This means that complex approximation error (12) or (13) and its $N-1$ derivatives $\hat{E}^{(i)}(\omega)$, where $i=1,2, \ldots, N-1$ have to satisfy the following condition

$$
\hat{E}^{(i)}\left(\omega_{0}\right)=\hat{H}_{N}^{(i)}\left(\omega_{0}\right)-\hat{H}_{\mathrm{id}}^{(i)}\left(\omega_{0}\right)=0
$$

at frequency of maximal flatness, which in this case is $\omega_{0}=$ 0 . This means that derivatives of frequency responses of the designed FIR filter and the ideal filter have to be equal at $\omega_{0}$

$$
\hat{H}_{N}^{(i)}\left(\omega_{0}\right)=\hat{H}_{\mathrm{id}}^{(i)}\left(\omega_{0}\right)
$$

where the $i^{\text {th }}$ derivative of $\hat{H}_{N}(\omega)$

$$
\hat{H}_{N}^{(i)}\left(\omega_{0}\right)=(-j)^{i} \sum_{n=0}^{N-1} h[n]\left(n-\tau_{N}\right)^{i} e^{-j \omega_{0}\left(n-\tau_{N}\right)}
$$

This leads us to equation

$$
\sum_{n=0}^{N-1} h[n]\left(n-\tau_{N}\right)^{i} e^{-j \omega_{0}\left(n-\tau_{N}\right)}=j^{i} \hat{H}_{\mathrm{id}}^{(i)}\left(\omega_{0}\right)
$$

Since the derivative of $\hat{H}_{\mathrm{id}}\left(\omega_{0}\right)$ for FD filter is following

$$
\hat{H}_{F D}^{(i)}\left(\omega_{0}\right)=(-j)^{i} \varepsilon^{i} e^{-j \omega_{0} \varepsilon}
$$

the condition for $i$-th derivative (17), which we will call further the $i$-th order condition, can be expressed by the following equation

$$
\sum_{n=0}^{N-1}\left(n-\tau_{N}\right)^{i} e^{-j \omega_{0}\left(n-\tau_{N}\right)} h[n]=\varepsilon^{i} e^{-j \omega_{0} \varepsilon}
$$

For $\omega_{0}=0$, which is the case of the MF filter, the condition (19) simplifies to

$$
\sum_{n=0}^{N-1}\left(n-\tau_{N}\right)^{i} h[n]=\varepsilon^{i}
$$

To obtain the impulse response of the MF filter we need to solve a set of $N$ equations (20) with $i=0,1, \ldots, N-1$.

\section{DMF FILTER DESIGN}

The MF approach can be readily generalized. Instead of choosing $\omega_{0}=0$ for all conditions we can distribute these conditions over several different frequencies. Using such an approach, called the DMF method [1], [16], we select a set of $N$ independent equations based on conditions specified with (19) and for each $k=0,1, \ldots, N-1$ we need to specify a condition order $i_{k}$ and a frequency $\omega_{k}$ at which this condition is forced. This selection determines properties of the designed filter which will be further discussed in the next section.

For $\omega_{k}=0$ the condition (17) simplifies to equation

$$
\sum_{n=0}^{N-1}\left(n-\tau_{N}\right)^{i_{k}} h[n]=j^{i_{k}} \hat{H}_{\mathrm{id}}^{\left(i_{k}\right)}(0)
$$

Since frequency responses of filter with real coefficient is a Hermitian function, all the coefficients in the above equation are real. Nevertheless, in the DMF method frequencies $\omega_{k}$ in (19) are in general not equal to zero. Therefore coefficients of the equation (17) are complex valued, which increases numerical costs and allows for impulse responses with complex samples. In practice coefficients of the designed filter are expected to be real. In order to guarantee the real impulse response of the designed filter, for each condition with $\omega_{k} \neq 0$ there must be also the condition of the same order for frequency $-\omega_{k}$. Because frequency response of the filter with real impulse response is Hermitian even symmetric, its derivatives demonstrate the following properties

$$
H^{\left(i_{k}\right)}(-\omega)=\left\{\begin{aligned}
\operatorname{conj}\left(H^{\left(i_{k}\right)}(\omega)\right), & i_{k}=0,2,4, \ldots \\
-\operatorname{conj}\left(H^{\left(i_{k}\right)}(\omega)\right), & i_{k}=1,3,5, \ldots
\end{aligned}\right.
$$

where $\operatorname{conj}(x)$ means complex conjugate of $x$. Therefore, based on the Hermitian symmetry of the frequency response of the designed filter, two conditions for $\omega_{k}$ and $-\omega_{k}$, with 
complex formulas (17) can be combined into two formulas with real coefficients

$$
\begin{aligned}
& \sum_{n=0}^{N-1}\left(n-\tau_{N}\right)^{i_{k}} \cos \left(\omega_{k}\left(n-\tau_{N}\right)\right) h[n] \\
& =\frac{j^{i_{k}}}{2}\left(\hat{H}_{\mathrm{id}}^{\left(i_{k}\right)}\left(-\omega_{k}\right)+\hat{H}_{\mathrm{id}}^{\left(i_{k}\right)}\left(\omega_{k}\right)\right) \\
& =\left\{\begin{array}{l}
(-1)^{i_{k} / 2} \operatorname{Re}\left(H_{\mathrm{id}}^{\left(i_{k}\right)}\left(\omega_{k}\right)\right), i_{k}=0,2,4, \ldots \\
(-1)^{\frac{i_{k}+1}{2}} \operatorname{Im}\left(H_{\mathrm{id}}^{\left(i_{k}\right)}\left(\omega_{k}\right)\right), i_{k}=1,3,5, \ldots
\end{array}\right. \\
& \sum_{n=0}^{N-1}\left(n-\tau_{N}\right)^{i_{k}} \sin \left(\omega_{k}\left(n-\tau_{N}\right)\right) h[n] \\
& =\frac{j^{i_{k}-1}}{2}\left(\hat{H}_{\mathrm{id}}^{\left(i_{k}\right)}\left(-\omega_{k}\right)-\hat{H}_{\mathrm{id}}^{\left(i_{k}\right)}\left(\omega_{k}\right)\right) \\
& =\left\{\begin{array}{c}
-(-1)^{i_{k} / 2} \operatorname{Im}\left(H_{\mathrm{id}}^{\left(i_{k}\right)}\left(\omega_{k}\right)\right), i_{k}=0,2,4, \ldots \\
(-1)^{\frac{i_{k}+1}{2}} \operatorname{Re}\left(H_{\mathrm{id}}^{\left(i_{k}\right)}\left(\omega_{k}\right)\right), i_{k}=1,3,5, \ldots
\end{array}\right.
\end{aligned}
$$

which in case of the FD filter take the following form

$$
\begin{aligned}
& \sum_{n=0}^{N-1}\left(n-\tau_{N}\right)^{i_{k}} \cos \left(\omega_{k}\left(n-\tau_{N}\right)\right) h[n]=\varepsilon^{i_{k}} \cos \left(\omega_{k} \varepsilon\right) \\
& \sum_{n=0}^{N-1}\left(n-\tau_{N}\right)^{i_{k}} \sin \left(\omega_{k}\left(n-\tau_{N}\right)\right) h[n]=\varepsilon^{i_{k}} \sin \left(\omega_{k} \varepsilon\right)
\end{aligned}
$$

Now, to specify the DMF filter design problem we simply need to select $N$ linear equations. Alternatively, the design problem can be expressed using the following matrix equation

$$
\mathbf{P h}=\mathbf{p}
$$

which can be transformed into the formula for impulse response of the FD filter

$$
\mathbf{h}=\mathbf{P}^{-1} \mathbf{p}
$$

where matrix $\mathbf{P}^{-1}$ is the inverse of the square matrix $\mathbf{P}$. Elements of matrix $\mathbf{P}$ together with vector $\mathbf{p}$ can be formed based on coefficients of the equation (16).

In case of FD filter for condition with $\omega_{k}=0$ the $k$-th row of matrix $\mathbf{P}$ has elements

$$
P_{i_{k}+1, n+1}=\left(n-\tau_{N}\right)_{k}^{i} ; \quad n=0,1, \ldots, N-1
$$

For $\omega_{k} \neq 0$ and $\omega_{k+1}=-\omega_{k}$ the $k$-th and $k+1$-st rows of matrix $\mathbf{P}$ take the following form

$$
\begin{aligned}
& P_{i_{k}+1, n+1}=\left(n-\tau_{N}\right)^{i_{k}} \quad \cos \left(\omega_{k}\left(n-\tau_{N}\right)\right) \\
& P_{i_{k}+2, n+1}=\left(n-\tau_{N}\right)^{i_{k+1}} \sin \left(\omega_{k}\left(n-\tau_{N}\right)\right)
\end{aligned}
$$

Similarly for $\omega_{k}=0$ we obtain the following elements of vector $\mathbf{p}$

$$
p_{1, i_{k}+1}=\varepsilon_{k}^{i}
$$

which corresponds to (27) and

$$
\begin{aligned}
& p_{1, i_{k}+1}=\varepsilon^{i_{k}} \quad \cos \left(\omega_{k} \varepsilon\right) \\
& p_{1, i_{k}+2}=\varepsilon^{i_{k+1}} \sin \left(\omega_{k} \varepsilon\right)
\end{aligned}
$$

which corresponds to (28).

Let us notice that if we want to design a different filter the matrix $\mathbf{P}$ (or equivalently the left side of equations (23)) does not change and only coefficients of the vector $\mathbf{p}$ have to be updated according to derivatives of the required ideal frequency response. Let us now consider determination of vector $\mathbf{p}$ for the differentiator and RC/SRRC filters.

In case of the differentiator only the first derivative is nonzero

$$
H_{\mathrm{dif}}^{\left(i_{k}\right)}(\omega)= \begin{cases}j, & i_{k}=1 \\ 0, & i_{k}=2,3, \ldots\end{cases}
$$

This gives us from (23) a vector $\mathbf{p}$ with elements

$$
\begin{aligned}
& p_{1, i_{k}+1}=\left\{\begin{array}{r}
0 \text { for } i_{k}=0,2,3, \ldots \\
-1 \text { for } i_{k}=1
\end{array}\right. \\
& p_{1, i_{k}+2}=\left\{\begin{aligned}
-\omega_{k} \text { for } i_{k}=0 \\
0 \text { for } i_{k}=1,2, \ldots
\end{aligned}\right.
\end{aligned}
$$

For the RC/SRRC filter the first and second derivative is following

$$
H_{\mathrm{RC}}^{(1)}(\omega)=\left\{\begin{array}{l}
-\beta \sin \left(a \beta\left(\omega-\omega_{\mathrm{p}}\right)\right) \text { for } \omega_{\mathrm{p}}<\omega<\omega_{\mathrm{s}} \\
0 \text { for } 0 \leq \omega \leq \omega_{\mathrm{p}} \text { and } \omega_{\mathrm{s}} \leq \omega \leq \pi
\end{array}\right.
$$

and

$$
H_{\mathrm{RC}}^{(2)}(\omega)=\left\{\begin{array}{l}
-a \beta^{2} \cos \left(a \beta\left(\omega-\omega_{\mathrm{p}}\right)\right) \text { for } \omega_{\mathrm{p}}<\omega<\omega_{\mathrm{s}} \\
0 \text { for } 0 \leq \omega \leq \omega_{\mathrm{p}} \text { and } \omega_{\mathrm{s}} \leq \omega \leq \pi
\end{array}\right.
$$

where $a=1$ for RC filter and $a=2$ for SRRC filter.

This time vector $\mathbf{p}$ depends on frequency $\omega_{k}$. For $\omega_{k} \in$ $\left[0, \omega_{\mathrm{p}}\right]$ we obtain

$$
\begin{aligned}
& p_{1, i_{k}+1}=\left\{\begin{array}{l}
1 \text { for } i_{k}=0 \\
0 \text { for } i_{k}=1,2
\end{array}\right. \\
& p_{1, i_{k}+2}=0
\end{aligned}
$$

while for $\omega \in\left(\omega_{\mathrm{p}}, \omega_{\mathrm{s}}\right)$

$$
\begin{aligned}
& p_{1, i_{k}+1}= \begin{cases}\cos ^{a}\left(\beta\left(\omega_{k}-\omega_{\mathrm{p}}\right)\right) & \text { for } i_{k}=0 \\
0 & \text { for } i_{k}=1 \\
a \beta^{2} \cos \left(a \beta\left(\omega_{k}-\omega_{\mathrm{p}}\right)\right) & \text { for } i_{k}=2\end{cases} \\
& p_{1, i_{k}+2}= \begin{cases}0 & \text { for } i_{k}=0 \\
\beta \sin \left(a \beta\left(\omega_{k}-c\right)\right) & \text { for } i_{k}=1 \\
0 & \text { for } i_{k}=2\end{cases}
\end{aligned}
$$

and finally for $\omega_{k} \in\left[\omega_{\mathrm{s}}, \pi\right]$

$$
p_{1, i_{k}+1}=0 \quad \text { and } \quad p_{1, i_{k}+2}=0
$$

We have demonstrated in this section how the DMF method can be applied to the differentiator and RC/SRRC filters which are zero-phase filters, and to the FD filter with non-zero phase response. In some applications it might be more convenient to combine those two types of filters into one filter and design a special filter with a fractional delay. The DMF method allows us for direct design of such filters. To achieve this goal we only need to modify the zero-phase frequency response of the filter into which we want to introduce fractional delay (7). Next, we need to apply to such modified frequency response the same rules (23) in order to obtain vector $\mathbf{p}$. The main difficulty of this procedure is the derivation of derivatives of $H_{\mathrm{id}, \mathrm{FD}}(\omega)$. However, to solve this problem we only need derivatives of components of $H_{\mathrm{id}, \mathrm{FD}}(\omega)$ which have been presented earlier 


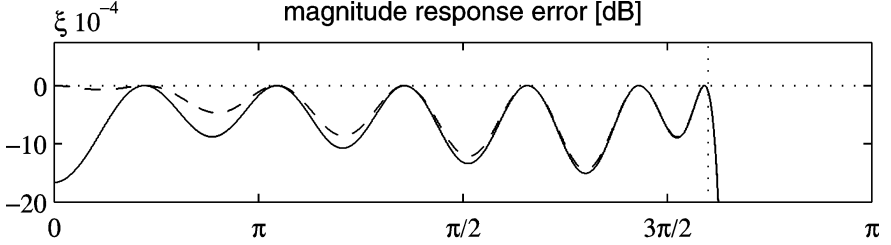

$\xi 10^{-3} \quad$ group delay error

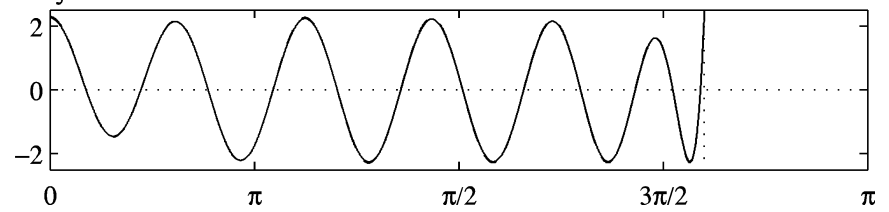

Fig. 1. Filter of the length $N=25$ with $f_{a}=0.4$ and $\varepsilon=-0.25$ with minimized magnitude response error. The case with zero order condition at $f=0$ - dashed line and with first order condition at $f=0$ - solid line.

in this section. For example first and second derivative can be computed using the following formulae

$$
\begin{aligned}
H_{\mathrm{id}, \mathrm{FD}}^{(1)}(\omega) & =H_{\mathrm{id}}^{(1)}(\omega) H_{\mathrm{FD}}(\omega)+H_{\mathrm{id}}(\omega) H_{\mathrm{FD}}^{(1)}(\omega) \\
H_{\mathrm{id}, \mathrm{FD}}^{(2)}(\omega) & =H_{\mathrm{id}}^{(2)}(\omega) H_{\mathrm{FD}}(\omega) \\
& +2 H_{\mathrm{id}}^{(1)}(\omega) H_{\mathrm{FD}}^{(1)}(\omega)+H_{\mathrm{id}}(\omega) H_{\mathrm{FD}}^{(2)}(\omega)
\end{aligned}
$$

Let us notice, that in most practical design problems we would only need these two derivatives.

\section{FD FILTER DESIGN WITH DMF}

The classic MF method [2] is a special case of DMF approach with $i_{k}=k$ and $\omega_{k}=0$. In paper [16] two other special cases are presented. First with zero order conditions $i_{k}=0$ used for all $N$ different frequencies $\omega_{k}$ which allows for design of a nearly optimal minimax filter. The frequencies $\omega_{k}$ must be placed at frequencies of local maxima of complex approximation error of the optimal minimax filter [16]. The second special case is the filter with passivity property like in case of MF filter but with user defined approximation band width. In [16] this case was limited only to filters of the even length $N$ with the complex approximation error equiripple in the approximation band. This case was extended to all filter lengths in [1]. To obtain this solution the zero and second order condition must be placed at every second frequency of local maxima of the magnitude of the complex approximation error (12) of the optimal minimax filter [16]. These examples confirm a huge potential of the DMF approach which allows for design of filters of different type. However, the efficiency of the proposed approach depends on the selected set of the frequencies $\omega_{k}$, therefore the important part of the method is the iterative algorithm for determination of the optimal $\omega_{k}$ frequencies.

Let us consider zero $\left(i_{k}=0\right)$ and first $\left(i_{k}=1\right)$ order conditions. With the zero order condition the approximation error $E(\omega)$ at $\omega_{k}$ is equal to zero. This means that at $\omega=\omega_{k}$ the magnitude response $\left|H_{N}(\omega)\right|=1$. The first order condition guaranties that the error $E(\omega)$ has local extremum at $\omega_{k}$. If this local extremum is actually the local maximum then zero and first order conditions forced at the same frequency guarantee that around $\omega=\omega_{a}$ the magnitude response is no greater than
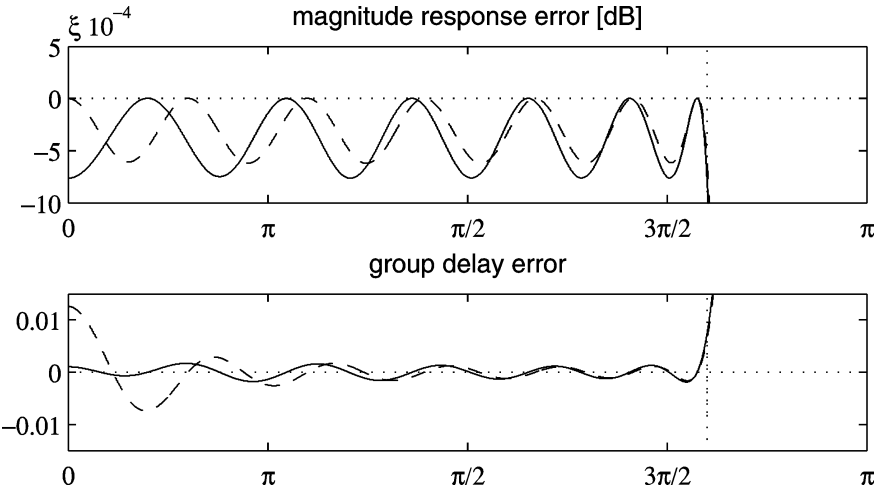

Fig. 2. Filter of the length $N=25$ with $\omega_{a}=0.8 \pi$ and $\varepsilon=-0.25$ with minimized group delay error. The case with zero order condition at $f=0$ dashed line and with first order condition at $f=0-$ solid line.

one. Additionally, the group delay (11) [16]

$$
\tau\left(\omega_{k}\right)=-\operatorname{Im}\left(\frac{-j \tau_{d} H_{\mathrm{id}}\left(\omega_{k}\right)}{H_{\mathrm{id}}\left(\omega_{k}\right)}\right)=\tau_{d}
$$

which means that the group delay error at $\omega=\omega_{a}$ is equal to zero.

Let us consider passive FD filters [1] which means that for each different $\omega_{k}$ at least zero and first order conditions need to be satisfied

$$
\omega_{k}=\omega_{k+1}: \quad i_{k}=0 \quad \text { and } \quad i_{k+1}=1
$$

This condition is necessary but not sufficient. The actual passiveness of the filter depends on all $N$ conditions selected for particular filter design.

In the paper [1] four cases, dependent on filter length $N$, which guarantee passive FD filter design at least for $D=\operatorname{round}((N-1) / 2)$ and $d \in[-0.5,0.5]$, have been proposed. The simplest cases are for even length filters since we simply need to select $N / 2$ different frequencies $\omega_{k}$ at which zero and first order conditions are forced (40). This gives as $N$ linear equations. The difference between even $N / 2$ (length of filter divisible by 4) and odd $N / 2$ is that for the latter case, one of the frequencies $\omega_{k}$ is placed at $\omega=0$. The odd length cases are more problematic since we need to define the odd number of conditions. For even $(N-1) / 2$ we propose that at zero frequency $\omega=0$ only zero or first order condition is set and the other one is omitted. Which variant is better, the one with the zero order condition or with the first order condition, depends on particular design case. In Fig. 1 the group delay of designed filters is the same for both variants but the magnitude response error is smaller when the first order condition is forced at $\omega=0$. We obtain a different situation when the magnitude response is minimized during design (Fig. 2). For first order condition the magnitude response error is again a bit smaller when compared to the filter with the zero order condition at $\omega=0$. Nevertheless, this improvement results in significantly larger group delay error around $\omega=0$. Finally, for $(N-1) / 2$ odd, apart from zero and first order conditions, the second order condition needs to be set at zero frequency. 

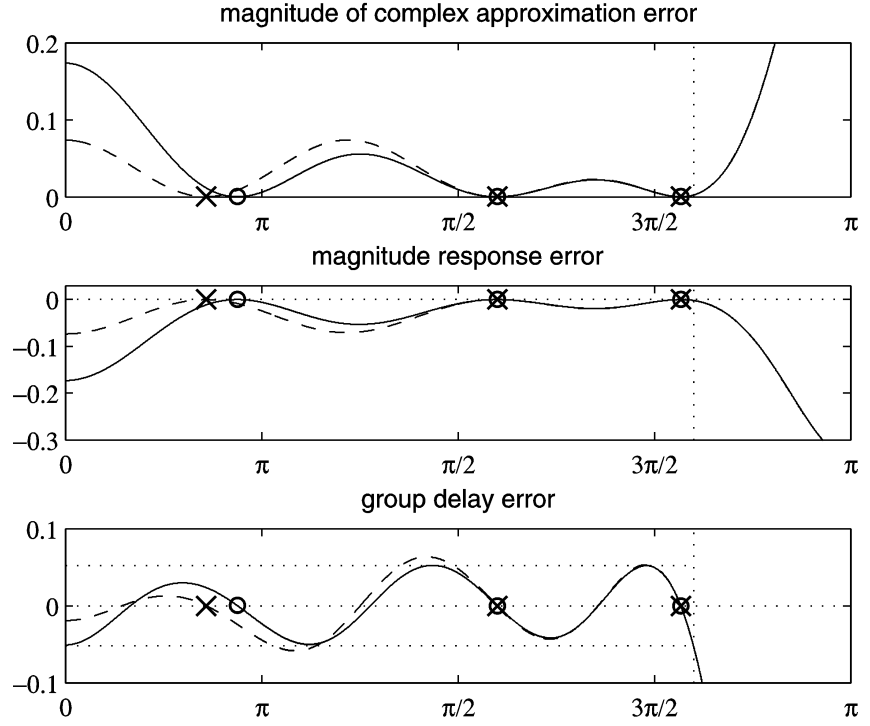

Fig. 3. Effects of manipulations on set of $\omega_{k}$ for filter of length $N=12$ with $\omega_{a}=0.8 \pi$ and $\varepsilon=0.25$. First set of $\omega_{k}$ marked with 'x', second with 'o'.

\section{ITERATIVE SEARCH FOR Optimal Frequencies of DMF CONDITIONS}

In previous section we have presented the general concept of the MF conditions distribution over the approximation band of the designed filter which ensure passiveness of the filter. In practice a designed filter needs to fulfill additional conditions like minimization of magnitude response or group delay error in the desired approximation band. In order to achieve such a goal a proper set of frequencies $\omega_{k}$ has to be found. In Fig. 3 frequency responses of two filters differing only in position of a single frequency point $\omega_{0}$ are presented. With frequency point moved towards zero the interval $\left[0, \omega_{0}\right]$ decreases and the interval $\left[\omega_{0}, \omega_{1}\right]$ increases. This results in decrease of the error of all three frequency responses presented in Fig. 3 in the first interval, while the errors increase in the second interval. This property can be used in iterative search for optimum set of frequencies $\omega_{k}$ with frequency responses presented in Fig. 3 selected as the error function. The three basic candidates for error function are magnitudes of (1) group delay error, (2) magnitude response error and (3) complex approximation error. Since the last two result in very similar designs, only first two will be presented in this paper. The designer can select different function but the basic criteria for error function is that the increase in distance between two consecutive frequency points $\omega_{k}$ must result in increase of the maximum of the error function located between those two frequencies.

We propose the following recursive algorithm for $\omega_{k}$ frequencies adjustment (Fig. 4) based on selected error function

1) select initial set of $\omega_{k}$ frequencies for $k=1, \ldots, N$, additionally including the edge frequencies of the approximation band $\omega_{0}=-\omega_{a}$ and $\omega_{N+1}=\omega_{a}$ not related to any of conditions $i_{k}$, e.g. uniformly spread the frequency points over the approximation band $\left[-\omega_{a}, \omega_{a}\right]$,

2) compute elements of matrix $\mathbf{P}$ and vector $\mathbf{p}(25)$,

3 ) compute the impulse response (26) and the corresponding error function,

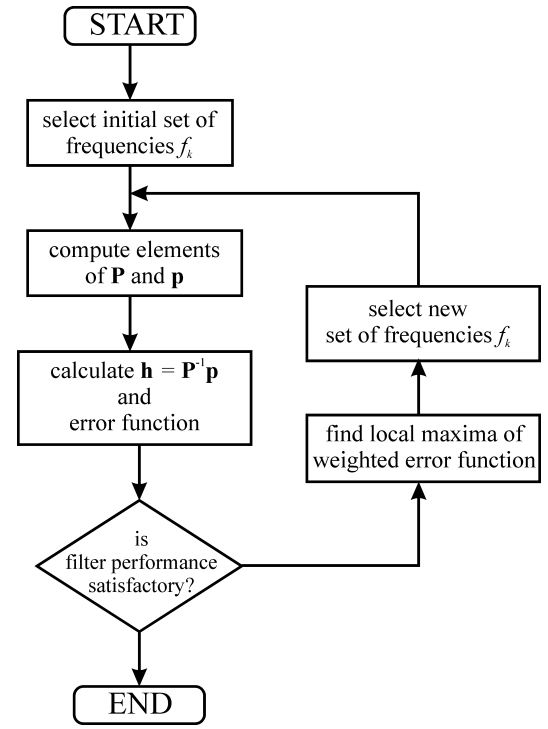

Fig. 4. Diagram of algorithm for searching the best frequencies $\omega_{k}$ of DMF conditions.

4) if the obtained filter satisfies performance requirements stop the algorithm,

5) multiply error function by the weighting function $W(\omega)>0$ and find maxima of the weighted function $e_{k}=\max _{\omega \in\left[\omega_{k-1}, \omega_{k}\right)}(W(\omega) E(\omega))$ for segments between each pair of consecutive frequencies $\omega_{k}$,

6) change distance between each pair of consecutive frequencies $\omega_{k}$ accordingly to weighted error maxima (41): move $\omega_{k}$ frequencies farther apart for larger maximum and closer for smaller maximum ((42) and (43)),

7) begin next iteration starting from point 2 .

To compute a set of new frequency points we need ordered frequencies $\omega_{k}\left(\omega_{k}<\omega_{k+1}\right.$ for $\left.k=1, \ldots, N-1\right)$ and values of local maxima $e_{k}$ of weighted error function. Using relative maximum errors

$$
\Delta e_{k}=\left(e_{k}-\min \left\{e_{k}\right\}\right) / \max \left\{e_{k}\right\}, \quad k=1, \ldots, N+1
$$

we propose the following formula for scaled frequencies

$$
\Delta \hat{\omega}_{k}=\left(1-\Delta e_{k}^{a} / b\right)\left(\omega_{k}-\omega_{k-1}\right)
$$

where parameters $a$ and $b$ determine the convergence rate and for FD filters $a=2$ and $b=10$ offer a good convergence rate and do not result in algorithm overdriving. Using (42) we can finally compute a set of new frequencies

$$
\hat{\omega}_{k}=\omega_{a}\left(2 \frac{\sum_{i=0}^{k} \Delta \hat{\omega}_{k}}{\sum_{i=0}^{N} \Delta \hat{\omega}_{k}}-1\right)
$$

It is worth noting that for given set of conditions $i_{k}$ and $\omega_{k}$ the matrix $\mathbf{P}$ ((27) and (28)) is independent of the required net delay $\varepsilon$. Additionally, for FD filters the search for optimum set of $\omega_{k}$ for different net delays $\varepsilon$ with all other conditions unchanged leads to the same set of frequencies $f_{k}$. This means that just like in the case of minimax FD filter design [9], [10], [16] the inverse matrix $\mathbf{P}^{-1}$ in (26) can be reused for different delays allowing for numerically efficient variable fractional delay (VFD) filter implementation. 

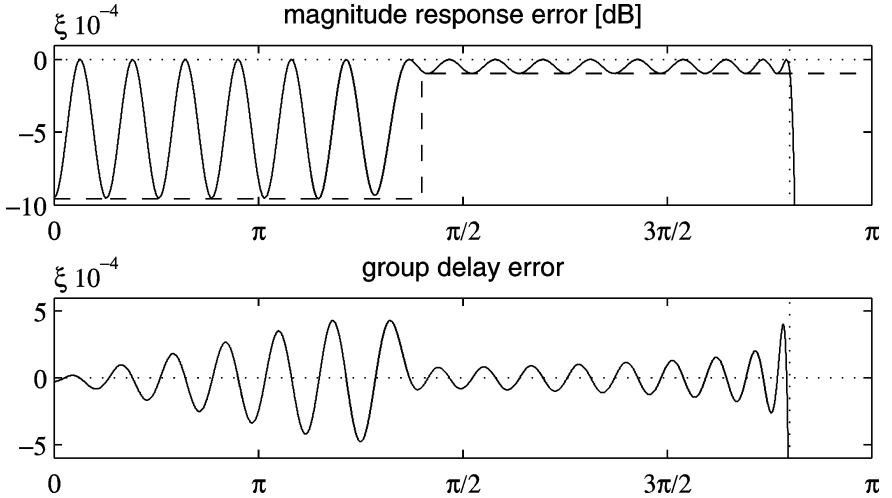

Fig. 5. FD filter of length $N=64$ with $\omega_{a}=0.9 \pi$ and $\varepsilon=0.25$ designed with weighted magnitude response error used as the error function. Dashed lines present group delay error limits resulting from weighting function.
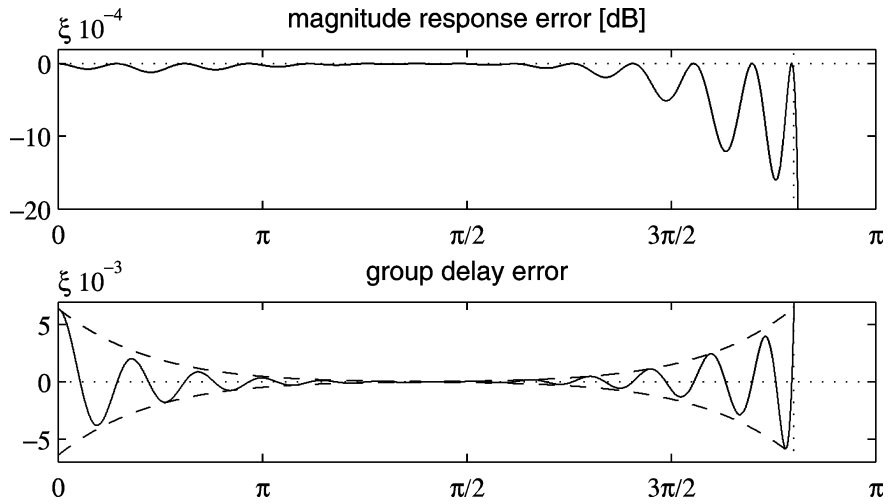

Fig. 6. FD filter of length $N=53$ with $\omega_{a}=0.9 \pi$ and $\varepsilon=-0.25$ designed with weighted magnitude of group delay error used as the error function. Dashed lines present group delay error limits resulting from weighting function.

\section{DESIGN EXAMPLES}

Figures 5 and 6 present two FD filters designed based on different error functions. As we can see both designed filters are passive, their magnitude response is no greater than one, in the whole frequency range. For the first filter the magnitude response was used as the error function, which results in equiripple magnitude response of the designed filter in each segment of weighting function. Such a filter has group delay with ripples increasing with frequency with smaller error for more strict magnitude response error weight. For the second filter the magnitude of group delay error was used as the error function. Also in this case conditions specified by the weighting function are met.

Figure 7 presents differentiators with non-zero fractional delay designed using the same procedure as FD filters. The first differentiator designed with only zero order conditions $\left(i_{k}=0\right)$ used, all at different frequencies. The second differentiator have been designed with pairs of zero and first order conditions forced at same frequency $\left(i_{k}=0, i_{k+1}=1\right.$ and $f_{k}=f_{k+1}$ ) just like in case of the passive FD filter. For the filter of odd length $N$ this results in magnitude response error no greater than zero, which might be useful if the filter has to be used in feedback loop. Nevertheless, at the same time the magnitude of complex approximation error is worse by $3 \mathrm{~dB}$ in comparison to the first filter. It is worth noting that magnitude response
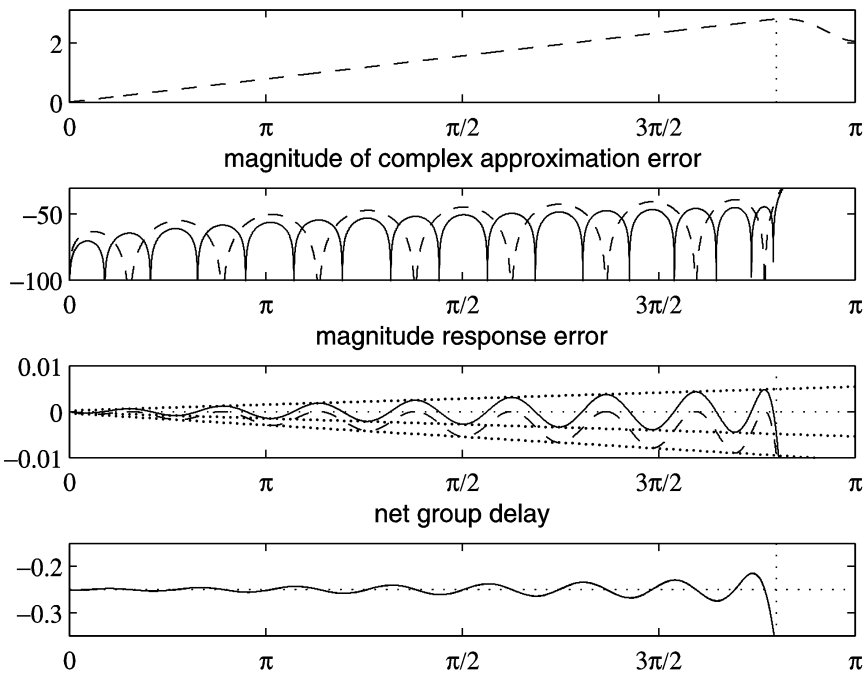

Fig. 7. Differentiators of length $N=33$ with $\omega_{a}=0.9 \pi$ and $\varepsilon=-0.25$ designed with weighted magnitude response error used as the error function. Solid lines - the case with only zero order conditions $\left(i_{k}=0\right)$ and dashed lines - the case with zero and first order conditions $\left(i_{k}=0,1\right)$

group delay error is the same for both filters. In both cases the weighting function linearly proportional to the frequency have been used, which for the differentiator results in constant relative error.

The same design procedure can also be used to design RC/SRRC filters with non-zero fractional delay. Figure 8 presents the example of such RC filter. The advantage of the proposed DMF method in case of RC/SRRC filter design is that the optimization can be performed in the whole frequency range including the transition band. This is important since the shape of frequency response in the transition band is important for Nyquist filters. As we can see in Fig. 8 the magnitude response error is smaller than or equal to zero in passband and transition band.

Let us notice that for both the differentiator and RC/SRRC filters the use of the group delay as the error function is difficult. This results from group delay error becoming very large around $\omega=0$ for differentiators of even length and at stopband for RC/SRRC filters. The design based on group delay error can be implemented but it requires algorithm modification in which a special care would be taken in the aforementioned regions. Another difficulty results from piecewise definition of RC/SRRC filter frequency response which means that derivatives of the error function are discontinuous. This affects algorithm convergence if the frequency $f_{k}$ of the condition needs to cross such discontinuity. To solve this problem we can optimize frequencies $f_{k}$ separately in each region and after reaching local optimum change a number of conditions in each region, moving points from regions with too low error to regions with too high error, and next repeating the optimization.

Finally, in Figs. 9 and 10 examples of the differentiator and SRRC VFD filters are presented. A single filter with arbitrary selected delay (here $\varepsilon=0.25$ ) was designed beforehand and later used with precomputed set of frequencies $\omega_{k}$ and the inverted matrix $\mathbf{P}^{-1}$ to design a family of filters with different 
magnitude response
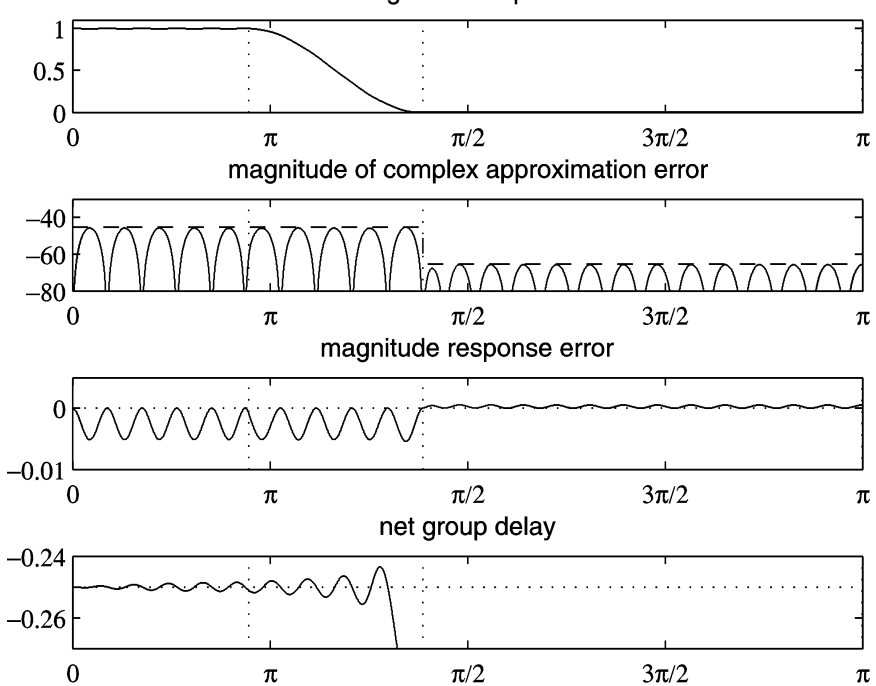

Fig. 8. RC filter of length $N=94, L=3, \alpha=0.33$ and $\varepsilon=-0.25$ with fullband optimization. Filter designed with weighted magnitude of complex approximation error used as the error function. Dashed lines represent error limits resulting from weighting function.
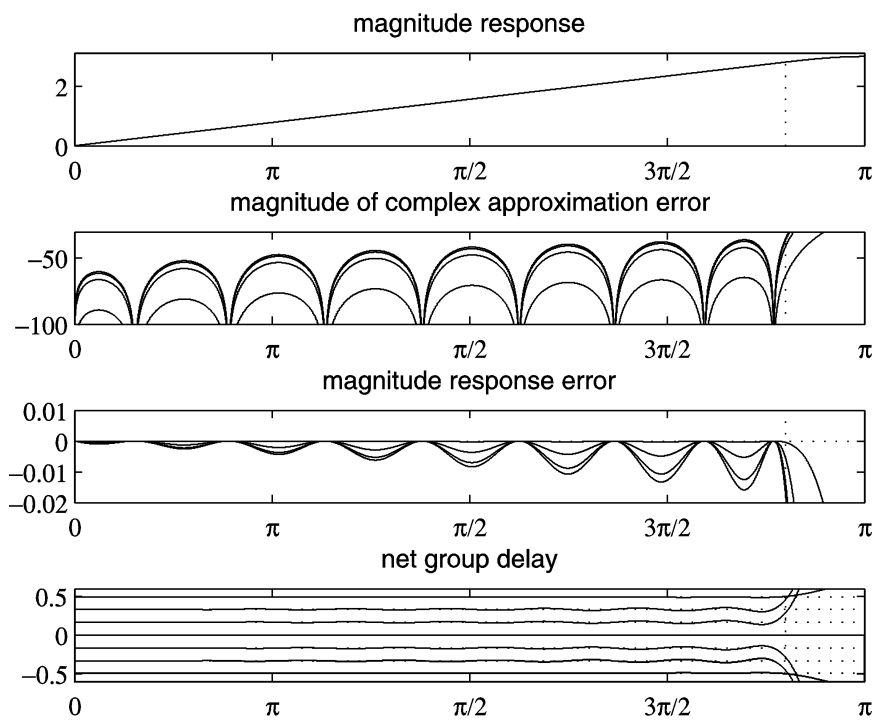

Fig. 9. Set of differentiators of length $N=33$ with $\omega_{a}=0.9 \pi$ (Fig. 7) for seven net delays $\varepsilon$ uniformly spread over the range $[-0.5,0.5]$.

fractional delays. For the differentiator, similarly to the FD filter, the error decreases for fractional delay $d$ close to zero. Also exactly like in case of VFD filters [1] using the same set of optimum frequencies of conditions $f_{k}$ results in filters with the same properties (Fig. 9). For SRRC filter (Fig. 10) the optimum set of frequencies $f_{k}$ slightly changes with the net delay $\varepsilon$ which results in slight changes of properties of frequency response error, though the maximum error is similar for different fractional delays. Additionally, also in this case the magnitude response error in passband and transition band is no larger that zero for all delays.
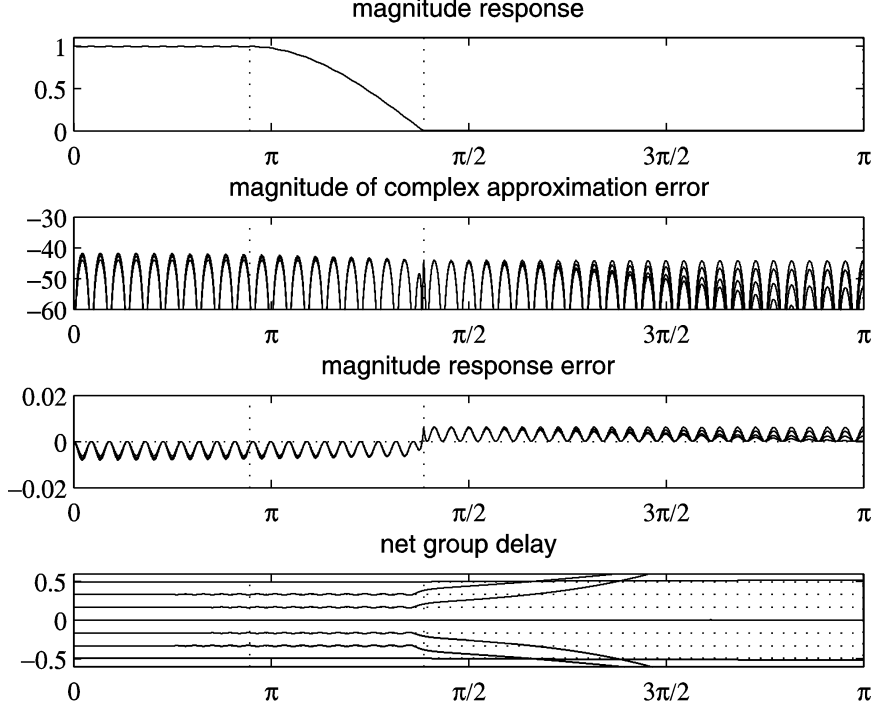

Fig. 10. Set of SRRC filters of length $N=178, L=3, \alpha=0.33$ with fullband optimization for seven net delays $\varepsilon$ uniformly spread over the range $[-0.5,0.5]$.

\section{CONCLUSION}

In this paper a filter design using DMF approach has been presented. The DMF method is the generalization of MF FD filter design procedure with conditions for the complex approximation error spread over the specified approximation band. We have demonstrated that with the proper selection of conditions designed FD filters are passive just like MF filters. The improvement over MF filters is that we can use the weighting function and the weighted error is optimized in the wider approximation band instead of best approximation only around $\omega=0$. Additionally, since the set of optimal frequencies $\omega_{k}$ is not dependent on fractional delay a passive VFD filter can be implemented with iterative search for $\omega_{k}$ and matrix $\mathbf{P}$ inversion done beforehand. Additionally, it has been demonstrated that the same approach can be used to design other filters. Examples for the differentiator and RC/SRRC filters with fractional delay have been presented. Since the properties of the designed filter depend on the selection of conditions orders and their locations, further investigations are planed on improved search algorithm for optimal set of $f_{k}$ frequencies for the differentiator and RC/SRRC filters, especially for the use with group delay error as the error function.

\section{REFERENCES}

[1] M. Blok, "Passive variable fractional delay filter design using distributed maximal flatness method," in Proc. ICSES'2012, 2012.

[2] T. I. Laakso, V. Välimäki, M. Karjalainen, and U. K. Laine, "Splitting the unit delay - tools for fractional delay filter design," IEEE Signal Processing Magazine, vol. 13, no. 1, pp. 30-60, 1996.

[3] E. Hermanowicz, Special descrete-time filters and applications. EXIT, 2005.

[4] C.-C. Tseng, "Designs of fractional delay filter, Nyquist filter, lowpass filter and diamond-shaped filter," Signal Process., vol. 87, pp. 584-601, 2007.

[5] S.-C. Pei and Y. Lai, "Closed form variable fractional time delay using FFT," IEEE Signal Process. Lett., vol. 19, no. 5, pp. 299-302, May 2012. 
[6] A. Yardim, G. D. Cain, and A. Lavergne, "Performance of fractionaldelay filters using optimal offset windows," in Proc. ICASSP'97, vol. 3 , Apr. 21-24, 1997, pp. 2233-2236.

[7] A. Yardim, G. D. Cain, and P. Henry, "Optimal two-term offset windowing for fractional delay," Electron. Lett., vol. 32, no. 6, pp. 526-527, Mar. 1996.

[8] T. I. Laakso, T. Saramäki, and G. D. Cain, "Asymmetric DolphChebyshev, Saramäki, and transitional windows for fractional delay FIR filter design," in Proc. MWSCAS'95, Aug. 1995, pp. 580 - 583.

[9] L. J. Karam and J. H. McClellan, "Complex Chebyshev approximation for FIR filter design," IEEE Trans. Circuits Syst. II, vol. 42, no. 3, pp. 207-216, Mar. 1995.

[10] M. Blok, "Optimal fractional sample delay filter with variable delay," in OSEE 2002, TechOnLine, Bedford, Massachusetts, USA, Mar. 18, 2002. [Online]. Available: http://www.eetimes.com/design/analogdesign/4018005

[11] P. J. Kootsookos and R. C. Williamson, "FIR approximation of fractional sample delay systems," IEEE Transactions on Circuits and Systems II: Analog and Digital Signal Processing, vol. 43, no. 3, pp. 269-271, Mar. 1996.
[12] E. Hermanowicz, "Explicit formulas for weighting coefficients of maximally flat tunable FIR delayers," Electron. Lett., vol. 28, no. 20, pp. 1936-1937, Sep. 1992

[13] A. G. Dempster and N. P. Murphy, "Lagrange interpolator filters and binomial windows," Signal Process., vol. 76, no. 1, pp. 81-91, Jul. 1999.

[14] M. M. Jahani Yekta, "A frequency domain proof for the equivalence of the maximally flat FIR fractional delay filter and the Lagrange interpolator," Digit. Signal Process., vol. 21, no. 1, pp. 13-16, Sep. 1992.

[15] V. Välimäki, "Discrete-time modeling of acoustic tubes using fractional delay filters," Ph.D. dissertation, Helsinki University of Technology, Faculty of Electrical Engineering (now Department of Electrical and Communications Engineering), Laboratory of Acoustics and Audio Signal Processing, Dec. 181995.

[16] M. Blok, M. Rojewski, and A. Matyjas, "Fractional delay filter design with non-uniform frequency sampling in frequency domain," Zeszyty Naukowe Wydziatu ETI PG, vol. 9, pp. 159-168, 2006, in Polish. 